Cultures \& Conflits

03 | automne 1991

Mafia, drogue et politique

\title{
Signe du religieux, conflits de société et connivences étatiques
}

Odon Vallet

\section{(2) OpenEdition \\ Journals}

Édition électronique

URL : http://journals.openedition.org/conflits/119

DOI : $10.4000 /$ conflits. 119

ISSN : 1777-5345

Éditeur :

CCLS - Centre d'études sur les conflits lilberté et sécurité, L'Harmattan

Édition imprimée

Date de publication : 17 octobre 1991

ISSN : 1157-996X

Référence électronique

Odon Vallet, "Signe du religieux, conflits de société et connivences étatiques », Cultures \& Conflits [En ligne], 03 | automne 1991, mis en ligne le 31 décembre 2002, consulté le 30 mars 2021. URL : http:// journals.openedition.org/conflits/119; DOI : https://doi.org/10.4000/conflits.119

Ce document a été généré automatiquement le 30 mars 2021.

Creative Commons License 


\title{
Signe du religieux, conflits de société et connivences étatiques
}

\author{
Odon Vallet
}

1 Le retour des signes religieux dans les sociétés européennes est régulièrement objet de polémique. Foulard dit islamique, minarets, ramadan, nourriture kasher ou hallal posent le problème des liens visibles entre coreligionnaires dès lors que les confessions sont visibles et leurs observances contradictoires. La dimension conflictuelle de ces prescriptions est primordiale lorsque les limites des nations ne recoupent pas les clivages religieux. L'exemple de la France est particulièrement significatif en ce que l'ancienne "fille aînée de l'Église" est aussi un carrefour des trois grandes filiations du monothéisme.

Les signes vestimentaires

2 Un premier groupe de signes concerne la parure ou le vêtement comme "deuxième peau" et habillage des convictions. Le principal conflit a concerné ce foulard qu'on veut à tout prix confondre avec l'Islam même s'il est déjà attesté dans les tablettes assyriennes $(\mathrm{A}, 40)$ de Téglatphalesar Ier, deux mille ans avant Mahomet. Pourquoi cette confusion? D'un point de vue musulman rigoureux, on n'aime guère évoquer les apports de la période antéislamique, cette Jahiliya qui est, dans la langue arabe, dérivée de l'ignorance, de la sottise et de la passivité. On ne voudrait pas confier l'honneur des femmes à un accessoire païen attesté par des tablettes impies et les lois assyriennes sont aussi suspectes que les légendes pharaoniques : les épines des cunéiformes ou les oiseaux des pictogrammes semblent receler quelque dangereux mystère, quelque blasphème en hiéroglyphes que seule efface la perfection des arabesques, cet art de mettre en courbes la droiture de la loi. D'un point de vue étroitement occidental, on souhaite oublier que le christianisme a intégré, comme l'Islam, les coutumes du ProcheOrient. Elles sont déjà présentes chez Isaïe $(3,17)$ pour qui Dieu découvre le front des femmes coquettes comme par un châtiment qui rend galeux leur crâne. Elles persistent chez saint Paul (I, Corinthiens 11,6) pour qui la femme doit prier tête couverte : Si la femme ne porte pas de voile, qu'elle se fasse tondre". Et le sens premier du mot "voile" 
en français (XIIème siècle), c'était le grand rideau du temple de Jérusalem ou, plus généralement, toute étoffe masquant le visage d'un dieu.

Plus encore, l'affaire du voile nous rappelle la chevelure de la pécheresse qui essuie les pieds de Jésus avec ses cheveux (Luc, 7,38). Pour les Assyriens, les femmes mariées doivent porter le voile, mais les prostituées voilées recevront cinquante coups de bâton et on leur versera de la poix sur la tête. Quel modèle choisir entre la Loi qui impose le voile et Jésus qui pardonne aux cheveux ? Il n'y a plus guère qu'aux audiences de JeanPaul II ou aux messes intégristes que les femmes portent la mantille. Les fidèles femmes nue-tête de nos églises seraient-elles donc pécheresses? Enfin, l'affaire du voile nous rappelle le temps où les bonnes soeurs portaient cornette, où le Bon Marché vendait tous les modèles de voile et de col empesé, où infirmières et veuves de guerre étalaient leurs voilages dans la rue, où chaque famille chrétienne avait sa religieuse invitant tous les siens à la cérémonie de prise de voile.

4 Faut-il donc oublier un passé si proche ? Le crâne rasé des adeptes de Krishnah est-il plus incongru que celui des disciples de saint Benoît? Naguère, les séminaristes débutants aimaient exhiber leur impeccable tonsure. A l'inverse, la pilosité des islamistes évoque la barbe généreuse des missionnaires d'autrefois, du plus bel effet sur le montant de la quête. Mi-religieuse, mi-nationale, on peut encore citer la kiffieh palestinienne, pas plus ridicule que le béret basque comme couvre-chef Ce qu'il y a peut-être d'insupportable dans ce signal de la vêture, c'est que des étrangers nous renvoient à la proximité de nos archaïsmes.

5 L'autre malaise provient sans doute du rappel des uniformes. Si toutes les filles d'un collège viennent avec le foulard, elles évoquent le temps du fichu des orphelins, du col blanc des pensionnats. Ce n'est qu'en mai 68 que les lycéennes de province ont quitté la blouse bleue et les instituteurs la blouse grise.

On retrouve ici le rôle unificateur des religions. Que la religion soit cueillette (lego) comme le pensait Cicéron ou lien (ligo) selon l'étymologie de Tertullien ou de Lactance, elle est toujours rassemblement: recueil de textes épars (épîtres ou sourates) et recueillement de l'esprit dispersé, relecture de préceptes divers (torah ou charia) et reliure de missels débordants. La religion est à l'origine du livre (codex) et, à Cordoue comme à Limoges, les relieurs ont tanné le cuir pour réunir les mots de Dieu.

7 On peut dire que cette réunion est arrachement car toute nouvelle religion ou tout renouveau religieux prend ses fidèles chez les exclus. Elle délie d'anciens engagements pour rénover d'autres obligations. Mais au sein de l'ensemble ainsi formé, il n'y a pas place pour la différence, l'hétérodoxie supposant un réseau contradictoire d'attachements. La contrainte de l'uniforme est une garantie de cohésion, une assurance contre l'éclatement, au moins à l'échelle du quartier. Le costume noir des juifs hassidim de Brooklyn est un défi aux baskets multicolores des gamins de New York, dans cette ville de shoe crime où l'on tue pour une paire de Nike.

Les rituels diététiques

8 La France est particulièrement sensible à ce rôle unifiant de la religion et de ses signes dans la mesure où le catholicisme fut la confession dominante. Depuis qu'il a perdu ce rôle, la France s'inspire du modèle américain multi-religieux de spiritualité concurrentielle et d'observances antagonistes (rebirth ou New Age) par la rupture et la secte retrouve son double sens et sa confusion première de coupure (Seco) et de suivisme (Sequor). 
9 Un bon exemple en est donné par les préceptes alimentaires. A peine a-t-on oublié le Carême que voici le Ramadan. Si l'un se substituait à l'autre, il n'y aurait qu'une conversion de la France au sein du même système pénitentiel, le jeune et le prêche passant de quarante jours au temps d'une lunaison et les joies de Carnaval se muant en ram'dam.

10 Mais, l'on prononce encore des sermons à Notre-Dame tandis qu'on programme à la télévision une nuit du ramadan. On fait maigre les vendredis de Carême dans les écoles publiques tandis qu'on y évite de plus en plus le porc, naguère délice des cantiniers impécunieux. Ici encore, comme aux Etats-Unis, on se rapproche du modèle éclaté des gastronomies substantielles où chacun cherche à se restaurer de traditions tout en se nourrissant d'exotisme à travers le kasher chinois ou le hallal indien.

11 C'est au temps du fast-food uniforme que la multiplicité des religions vient introduire de la variété dans la cuisine, rappeler ce temps où chaque groupe humain savait donner un peu d'épice à l'existence, ail et poivron du midi, girofle et cumin du nord-est. Ce temps où il y avait un lipide pour chaque région : beurre salé de Bretagne, saindoux de Lorraine, graisse d'oie de Gascogne ou huile d'olive de Provence. Un temps de diététique provinciale, identique pour tous les membres de la communauté, inconnue des étrangers.

Les liturgies pénitentielles

12 Lorsqu'elles tendent à introduire des distinctions en refusant le fait majoritaire, toute obédience est un schisme, toute observance une scission. Mais le séparatisme social peut servir la santé mentale lorsqu'il permet à l'individu de "retrouver ses racines", c'est-à-dire tout ce qui le sépare du tronc commun d'un mode de vie banalisé. C'est parfois pour éviter d'être schizophrène qu'on devient schismatique. Et la communauté juive, naguère si présente dans la psychanalyse, s'est aujourd'hui davantage tournée vers la religion, quittant ce qui dissout les malaises pour ce qui fédère les énergies. Woody Allen symbolise cette cure interminable qui pousse ses cadets à choisir d'autres voies d'apaisement. Chacun trouvera la paix dans des rites, les uns, en battant leur coulpe au mur des Lamentations, d'autres en avouant leurs fautes dans des réunions charismatiques, d'autres en participant à des cérémonies pénitentielles sur les routes de pèlerinage, d'autres en criant leurs péchés dans les shows télévisés des Églises par satellite. Mais la culpabilité a besoin de visible pour s'évacuer comme en témoigne le déclin de la confession auriculaire.

Le semainier traditionnel

13 On pourrait en dire autant de l'enthousiasme, délire sacré contre les idées noires, fermeté de l'amen face au doute philosophique ou secours du hosannah devant les idéologies du soupçon. Les reportages insistent souvent sur la joie des "frérots" le rayonnement des convertis par rapport aux doctrines confinées, réduites à la défensive ou contraintes par leurs exigences : le divan est ingrat, la cellule est étroite. Il faut mettre le mieux-vivre au grand air et les photos ne manquent pas de la prière du Vendredi dans les rues de Marseille ou du Chemin de Croix sur la colline de Montmartre. C'est à la religion de mettre un peu de couleur dans ces villes tentaculaires où il y a cent ans déjà, Emile Verhaeren voyait $\mathrm{H}$ leurs murs noirs durant des lieues, immensément par les banlieues".

14 C'est aussi à la religion de casser le temps, de rompre le charme des jours ouvrables lorsque le calendrier républicain a quelque peu jauni. Maintenant que décline le muguet communiste du Premier mai ou les gerbes municipales du Onze novembre, il 
faut trouver d'autres jours du souvenir. Maintenant que se répand le modèle newyorkais des magasins ouverts sept jours sur sept, il faut inventer de nouveaux jours chômés, d'autres occasions pour les habits du dimanche.

Nouvel an orthodoxe, Pâque juive ou Lendemain du Sacrifice deviennent autant de commémorations qui donnent à une ville cosmopolite comme Sarcelles, pourtant symbole de l'uniformité, des allures de festival. On mesure la distance par rapport au Jour de fête de Tati, sa bicyclette et son accordéon qui faisait danser tout un village au même pas. Désormais, la fête change à chaque étage, elle se déploie dans la promiscuité et, s'il faut un peu d'effort pour donner une dimension ludique à la cage d'escalier, on doit convenir que la célébration trompe l'ennui et qu'il y a plus d'un rapport entre le retour des fêtes religieuses et la disparition des cinémas de quartier.

Les phares sur la ville

C'est encore à la religion de marquer la ville. La science laïque toisait les monuments de la foi lorsque la tour Eiffel détrôna les flèches de Cologne comme plus haut édifice au monde. Désormais la mosquée de Casablanca (175 mètres) dépasse la cathédrale rhénane et sa voisine d'Ulm au livre des records et, un peu partout, on assiste au renouveau des minarets. Péguy saluait de Chartres "la flèche unique au monde" et Mitterrand fit d'un clocher nivernais le symbole de la force tranquille, comme si toutes les familles spirituelles se retrouvaient dans cette maison commune de l'ecclesia (église). Ces retrouvailles de la communauté, les Musulmans souhaitent la fêter dans des lieux de prière dont la dimension verticale s'inscrit par les minarets comme naguère les campaniles dans les villes italiennes.

17 La hauteur pose souvent problème au regard des plans d'urbanisme, plus indulgents pour les tours de bureaux que pour les repères de la foi. La réaction conciliaire contre "l'Église triomphante" avait conduit les chrétiens à construire petit, à tapir des chapelles au pied des grands ensembles, à enfouir des oratoires dans les sous-sols de supermarchés comme dans ces villes américaines où les cathédrales semblent implorer les gratte-ciel.

18 "Faut-il transformer les églises en musées" se demandait, en 1975, un jésuite dans les Études. Le Corbusier, qui avait son atelier dans un immeuble de la Société de Jésus, construisait à Ronchamp une chapelle et à Évreux un couvent sans que transparaissent dans les formes la présence d'un pouvoir, cette alliance du trône et de l'autel qui faisait l'orgueil des basiliques .

19 Les intégristes accusèrent le clergé de transformer les églises en garage : comment convaincre les foules si l'on n'est plus porteur de signe, c'est-à-dire sémaphore ? Et voici que la concurrence des minarets redonne aux évêques le sens du grandiose et qu'à côté de la mosquée d'Evry pousse une cathédrale, par ailleurs trop dépourvue de voûtes et de flèches pour emporter l'adhésion des fidèles et la générosité des offrandes.

20 Peut-être va-t-on vers un renouveau du visible dans l'expression des croyances, peutêtre aussi un pastiche de l'ancien dans le retour du religieux. Les temps conciliaires d'aggiornamento étant révolus, et avec eux l'ouverture à la modernité, on assisterait à une réaction des valeurs sûres et des styles confirmés. Les époques du "néo" (néoromans, néo-gothique, néo-byzantin) ont correspondu à une restauration des vieux rituels (chant grégorien, motets polyphoniques) bien rassurant devant la montée des "horreurs" de l'art moderne qui, de Monet à Debussy imposait ses barbouillages et ses dissonances. L'inauguration quasi simultanée de la basilique néo-renaissance de 
Yamoussoukro et de la cathédrale néo-gothique de Washington illustrent cette stratégie sécurisante pour confirmer les fidèles.

II se produit alors une connivence des ecclésiastiques et des politiciens pour rassembler les foules par des disciplines liturgiques. Qu'il s'agisse des cérémonies orthodoxes et du mouvement russe Pamiat ou des messes en latin et du Front national français, la manifestation religieuse traditionnelle est perçue comme un réflexe identitaire qui, en prohibant l'indifférence, pousse à prendre parti.

Le poids des nostalgies

22 Face à ce retour impressionnant des édifices spirituels et des symboles religieux, deux attitudes symétriques semblent dominer : l'indignation des laïques, et l'indulgence des nostalgiques. L'indignation des laïques se nourrit d'une mémoire de combat du temps des multiples recours contre les empiétements du clergé, lorsque les colonnes du recueil Lebon témoignaient du contentieux des processions et sonneries de cloche. Une époque pourtant ambiguë de prénoms républicains et de Saint-Charlemagne où les "saints laïques" fondent "l'école des Sans-Dieu".

Pour les indulgents, la nostalgie retrouve ici son sens premier d'un mal du pays, d'un regret d'autrefois où le recteur de l'Ile de Sein régnait sur ses pêcheurs comme le maire laïque de Clochemerle sur ses vignerons, où la politique anticléricale masquait la "sainte alliance des possédants": cette "manoeuvre de la classe capitaliste pour détourner les travailleurs de leur lutte contre la servitude économique"(1).

La nostalgie est aussi celle d'un temps d'Union sacrée, des "curés sac au dos" et des capitaines-instituteurs, un temps où, pour trouver du travail, il fallait son billet de confession ou son diplôme de bon soldat. Indignés et indulgents trouveraient alors leur synthèse dans le projet d'un renouveau concordataire où un Islam policé assurerait la paix des banlieues et un christianisme républicain la soumission du prophétisme. La "sagesse "des imams pendant la guerre du Golfe et l'appui de certains évêques à la "guerre juste" inaugure bien cette politique. S'éloigne ainsi le spectre des musulmans alliés aux "États-terroristes" et des chrétiens gainés au pacifisme du Larzac. Le retour à la religion visible est aussi celui du clergé qui mobilise alors que l'époque de la religion "enfouie" était celle des idées qui fermentent.

Ce passage du militant au militaire n'est pas sans rappeler la gloire de l'Empire français avec ses trente millions de musulmans et la loyauté des spahis contre les uhlans. Ou encore le temps des religieux brancardiers de Teilhard de Chardin au fort de Douaumont. Un temps qui ramène aux origines du prêtre de la Bible, portant l'Arche de l'alliance au coeur de la bataille et les rouleaux de la Loi à la barbe des Infidèles.

Le refus du compromis, le réflexe identitaire fonde paradoxalement l'unité du pays en ce que tous les clergés sont d'accord pour mettre leurs liturgies au service de la cohésion nationale. S'il y a procession du Quinze août au pied du Panthéon, on consacre la France à la Vierge immaculée. Et si l'on dit le Coran dans la mosquée de Mantes, c'est aussi pour détourner de la drogue les jeunes des grands ensembles.

La contre-révolution de l'Etat clérical

Faut-il parler de paix sociale ou s'auto-discipline communautaire? On peut se demander s'il n'y a pas ici une mutation des rôles dans la canalisation des foules. Naguère, les rituels défilés du 1er mai tenaient lieu d'exutoire inoffensif aux rebelles en puissance et les réunions de cellule, de fantasmes conspirateurs aux exploités insoumis. Les actuelles manifestations de ferveur religieuse peuvent aussi traduire cette 
impuissance à changer le monde et ce besoin de substituts crédibles à l'illusion du progrès. Le retour des religions signe la fin des idéologies comme projets mobilisateurs mais marque le même souci des lendemains qui chantent. Le bicentenaire de la Révolution coïncide avec le déclin des idéologies, ce label qu'avait inventé, en 1796, le philosophe Destutt de Tracy. Il avait construit l'idéologie sur les débris de "l'ancienne métaphysique théologique". Le retour de celle-ci montre un renversement de perspective dans la gestion de l'espérance. La conjuration retrouve son sens premier: elle n'est plus promesse de soulèvement mais ligue de la prière.

Le clandestin passe des arrière-salles de café aux cryptes des églises, où se réunissent tant de groupes charismatiques. L'occulte passe des soviets aux chapitres et aux bolcheviks succède l'Opus Dei comme symbole manipulateur. Car le retour du religieux est un subtil équilibre de visible et d'invisible, de signes extérieurs et d'indices profonds qui attestent de sa vitalité.

Et c'est curieusement la république laïque qui finance ce retour du religieux comme si, au fond d'elle-même, elle ne se voyait d'autre avenir que dans une abjuration de l'incroyance. L'art est ici le premier support de cette conversion où l'on retrouve la traditionnelle confusion des typographes entre le culturel et le cultuel. Le meilleur exemple en est l'aide de l'État aux monuments historiques dont, en France comme en Europe, plus de la moitié sont des édifices religieux. Dans notre pays, plus de 600 millions de francs sont ainsi consacrés chaque année aux quinze mille bâtiments religieux protégés, sans préjudice des aides des collectivités locales aux édifices du culte, qu'ils soient ou non classés ou inscrits. II s'agit certes de maintenir un patrimoine mais aussi de restaurer une tradition avec tout ce que le mot "Pierre" évoque quant aux fondements de la chrétienté.

31 II semble fini le temps de la laïcité triomphante et de "la grande pitié des églises de France" (Barrès). Fini aussi le temps des voeux "Pour une Église servante et pauvre" (Congar), celui des "vicaires rouges" qui jetaient les prie-Dieu à la décharge. Désormais, l'imagerie sulpicienne est protégée au titre des beaux-arts et les chapelles bretonnes sont restaurées en vue des élections.

Ce revirement est d'ailleurs autant idéologique qu esthétique tant on ne peut séparer culte et culture. Le concile Vatican II avait marqué le retour des grandes époques iconoclastes où l'on renverse les statues pour briser les idoles, où l'on jette les icônes pour chasser des images. Ainsi dénonçait-on la mariolâtrie en remisant les Vierges à l'enfant dans les sacristies de campagne comme autrefois, les chastes Artémis dans les greniers des prosélytes. Mais aujourd'hui, les architectes des monuments historiques restaurent les objets du culte marial, ces supports de l'hyperdulie qui forment une bonne partie des cinquante mille objets mobiliers protégés par la loi. Et l'État réhabilite ce qu'au temps de l'impératrice Eugénie, on appelait les bondieuseries.

Le deuxième exemple est celui de l'enseignement catholique comme signe extérieur de la présence de l'Église dans le monde de l'éducation. Les 28,4 milliards de crédits étatiques (en 1991), en plus des subventions des collectivités locales, attestent de la collaboration de la puissance publique à cette oeuvre confessionnelle. Fini le temps de "l'école des corbeaux" prêchant le retour du roi et la fin de la "gueuse". Fini aussi le temps de la gauche chrétienne voyant dans les collèges huppés une perversion bourgeoise des valeurs évangéliques. Désormais, Église et État réunis promeuvent l'instruction d'un sixième des effectifs scolarisés sous la direction d'un appareil ecclésiastique. L'un des principaux problèmes est aujourd'hui de savoir si un tel 
système est extensible aux communautés juive et musulmane, et s'il faut accepter le modèle new-yorkais d'éducation séparée avec son risque d'écoles sectaires et d'enfants de ghettos.

Le ministre de l'Éducation nationale est soumis à des pressions croissantes pour qu'il facilite à la fois l'ouverture de nouveaux établissements confessionnels et l'enseignement de la religion à l'école publique. Derrière le problème de "l'heure du catéchisme" ou de l'extension des écoles sous contrat, se profile la question d'une société laïcisée par le week-end et la baisse de la pratique: les parents s'en remettraient aux éducateurs pour transmettre une foi qu'ils négligent et il reviendrait à l'État laïque de subventionner les confessions faute de proposer à la jeunesse d'autres valeurs visibles et "lisibles".

Le troisième exemple est la participation croissante de l'État et des collectivités locales au financement et au fonctionnement des instituts religieux de formation, à l'exception des facultés protestantes de théologie et en mettant de côté les cas particuliers de l'Alsace-Moselle. Qu'il s'agisse de formation pédagogique des maîtres de l'enseignement privé ou de fondation théologique des laïcs et religieux, les organismes concernés bénéficient des subventions de l'État pour leur fonctionnement matériel et de conventions avec des universités pour leur rayonnement intellectuel. II est significatif que cette collaboration se développe au moment de la disparition des Ecoles normales "temples" de la laïcité. Ici encore, il semble que l'État laïque délègue aux religions le soin de transmettre des valeurs alors qu'il se cantonnerait au rôle de soutien financier ou de régulateur juridique des promoteurs d'idéaux. On notera, à ce sujet, l'évolution contrastée des langues anciennes dites "mortes" mais fort vivantes dans les traditions religieuses.

D'un côté le latin et le grec ne sont plus étudiés respectivement que par $10 \%$ et $1 \%$ des bacheliers, contre $100 \%$ en 1900 . De l'autre, on observe un retour au latin et au grec chez les laïcs catholiques qui fréquentent désormais les facultés de théologie. De même, l'étude des langues orientales stagne dans l'enseignement supérieur public. Elle a même diminué par rapport à l'époque de l'école coloniale et des officiers des Affaires indigènes, et les récents regroupements des sections du CNRS ne la favorisent pas. Au contraire, l'étude de l'hébreu biblique et de l'arabe coranique se développe tandis que, faute de connaissances linguistiques suffisantes, les sciences des religions ne dépassent guère les limites de revues confidentielles. Que le sanscrit, qui passionnait Voltaire, soit confiné au rôle d'idiome de groupements d'extrême-droite en dit long sur le déclin culturel des langues dites sacrées. Et l'on n'oubliera pas que l'enseignement laïque est historiquement apparu lorsque les professeurs ont pu rivaliser avec les religieux en matière de langues et donc de sens. En ont-ils encore le projet et les outils?

Nos deux voies de l'Orient

Ce retour des signes du religieux dans le champ du social (auquel il faudrait ajouter les traces des sectes) est promesse de conflits dans la mesure où il n'y a plus identité de la religion du prince et du peuple, où s'affrontent des politiques et des théologies, où les différences spirituelles ne recouvrent pas les clivages sociaux. Peut-être aussi d'ailleurs, ces conflits peuvent-ils se neutraliser provisoirement s'il ne se dégage pas de nette majorité religieuse ou antireligieuse.

38 La fracture la plus évidente se situe aujourd'hui entre une civilisation "judéochrétienne" et une "immigration islamiste". Cette opposition est d'ailleurs bien sujette à caution tant le judéo-christianisme a été, dès le quatrième siècle, largement mis en 
minorité par le pagano- ou l'helléno-christianisme. Peut-être pourrait-on esquisser un autre regroupement autour d'un antisémitisme actif ou latent à condition d'étendre ce mot, apparu en pleine affaire Dreyfus, à tous les peuples de langue sémitique et d'abord aux Arabes volontiers confondus avec les musulmans. Si une opinion semble se dégager aujourd'hui, elle est d'abord négative et se manifeste sous la forme d'un front du refus de la religion importée. C'est une différence majeure avec l'anticléricalisme du début de ce siècle visant une religion catholique acclimatée depuis deux mille ans. La référence serait alors peut-être à chercher du côté du Proche-Orient antique et de la confrontation Juifs-Grecs.

Comme aujourd'hui, trois signes majeurs s'ajoutaient : la circoncision, la nourriture et le jour de congé. Le malaise était maximum dans ce lieu unitaire qu'est l'armée, notamment chez les mercenaires juifs des années grecques: plaisanteries sous la douche, révoltes à la cantine, problèmes de permission émaillaient la vie des garnisons. Le drame se précipita lors de la guerre si suggestive des Maccabées et s'acheva, sous la succession romaine, par le génocide de la guerre des Juifs, cette shoah tristement fondatrice de "l'Occident chrétien".

Mais peut-être une religion peut-elle en cacher une autre et faudrait-il chercher les germes de l'avenir du côté de l'Extrême-Orient, d'un néo-bouddhisme reformulé qui relancerait la guerre philosophique entre la Grèce et l'Inde, combattant la logique de la raison par la force de la prière. Une fois de plus, on citera Malraux et son XXIème siècle qui sera religieux ou ne sera pas". C'est un apocryphe tout comme son quasi-inverse soi-disant biblique et d'origine inconnue : "Aide-toi et le ciel t'aidera". L'avenir n'est écrit nulle part. C'est aux hommes de l'inventer.

\section{RÉSUMÉS}

Le " retour du religieux " est un acte de foi qui incarne l'âme dans des gestes. Quand les religions ou les sectes sont multiples, la pratique devient conflictuelle et engendre à son tour un appel à la laïcité comme gardienne de la cohésion sociale.

The " religious comeback " is an act of faith incarnating the soul in gestures. When religions or sects become numerous, their practice turns conflictive and generates an appeal to secularity as a guardian of social cohesion.

\section{INDEX}

Mots-clés : conflits, religion 
AUTEUR

ODON VALLET

Enseignant à Paris I et Paris VII 